\title{
An Efficient Packet Transmission Protocol Using Reinforcing Learning in Wireless Sensor Networks
}

\author{
Hyun Joo Park ${ }^{1}$, Seong Cheol Kim ${ }^{2}$ \\ ${ }^{1}$ Department of Smart Information and Telecommunication Engineering, \\ SangMyung University, 31066, Cheonan, Korea \\ ${ }^{2}$ Department of Convergence Electronics, SangMyung University, 20, Hongjimun \\ 2-gil, Jongro-gu, Seoul, Korea \\ cathy2369@smu.ac.kr;sckim@smu.ac.kr(correspondingauthor)
}

\begin{abstract}
The recently requested Medium Access Control (MAC) protocol in Wireless Sensor Network (WSN) is fast, reliable, and supports QoS. For this, this paper has proposed a data storage and efficient data transmission protocol using Reinforce learning based on priorities in order to increase the network performance. The suggested protocol not only stores data by applying the priority of data, but also guarantees priority transmission with various probabilities during transmission. The data to be transmitted are divided into Event Data and Normal Data according to their content, and stored in the transmission queue by priority using RLClassifier. In addition, in order to apply the priority to the data while they are stored in the queue, the transmission probability is applied differently. A comparison with QAML-MAC, which has been proposed to improve data storage and transmission efficiency, revealed that performance of transmission latency was improved about $17 \%$.
\end{abstract}

Keywords: MAC protocol, Priority aware, Reinforce-learning, Throughput, Quality of Service. 


\section{Introduction}

The application range of wireless sensor networks is wide. In a hyper-connected society, the quality of human life is improved in various ways. In the environmental area, it is used in the noise and air pollution detection, water pollution level measurement, and natural disaster management. In medical field, wireless sensor network is used in hospital patient management and remote medical support through home networks. Wireless sensor network is also used in many other fields that are difficult to enumerate, such as inventory management in logistics and distribution, toll collection in transportation, and telematics (Bertsekas and Gallager, 1987 and Sri Varsha and Naga, 2018). The studies on the various applications listed above are conducted under the premise that the connection and data collection, the two basic criteria of wireless sensor networks, basically operate according to human thinking (Ye, 2004). This focuses only on normal operation functions for data collection and storage, and is not suitable for situations which require customized data transmission based on collected data and discriminatory results on data properties in discussions on service quality (Yahya et al., 2010). It was reported that the quality of network service cannot be improved because normal data and event driven data are transmitted to the sink direction without distinction (Park et al., 2019). To-date, many studies on MAC protocol have focused on energy consumption in data transmission. However, depending on the application, QoS support still remains as an important problem to be solved (Zheng et al., 2017; Kim et al., 2019; Kim et al., 2018; Alfouzan et al., 2018).

In this paper, to improve QoS by classifying data transmission emergency and characteristics, we have proposed an algorithm that would help efficient transmission to improve the quality of service through application of reinforcement learning algorithm of artificial intelligence during storing data in the transmission queue. By applying the artificial intelligence theory that reflects the emergency and characteristics of data transmission to the queue storage algorithm, it is expected that the efficient transmission of data as well as energy saving at the node would be possible, and the life of the entire network may be prolonged (Daehyon, 2018 and Mononteliza, 2020). In order to improve the quality of network service, the collected data should have different transmission urgencies depending upon their characteristics. Then they are divided into normal transmission data with low priority and emergency data requiring emergency transmission with high priority (Park et al., 2019 and Zheng et al., 2017). Normal data are data that are regularly collected in the environmental field or have a measured value within a certain range (Park et al., 2019 and Park, 2011). If the measured value is observed within a pre-defined threshold value, its priority is low. Since it has the property of regular transmission, it is stored in the normal transmission queue. On the other hand, if the measured value lies out of the range or the degree of change is different from the generally observed change, then it is classified as Event Data and saved to the queue with high priority. 
The rest of the paper organized as follows. The related works are discussed in Section 2. In Section 3, the structure and operation method of RL-Classifier will be explained. In Section 4, performance comparison id made, and in Section 5, the conclusion of this paper is made.

\section{Related Works}

\subsection{EQ-MAC}

EQ-MAC (Park, 2011) was proposed for service differentiation among cluster-based protocols. Here QoS is supported through Classifier MAC and channel access MAC that classify traffic according to priority. However, EQ-MAC is basically only applicable to a one-hop structure between sensor nodes and head nodes. Therefore, it is difficult to apply to WSNs with multi-hop. EQ-MAC operation is shown in Fig. 1.

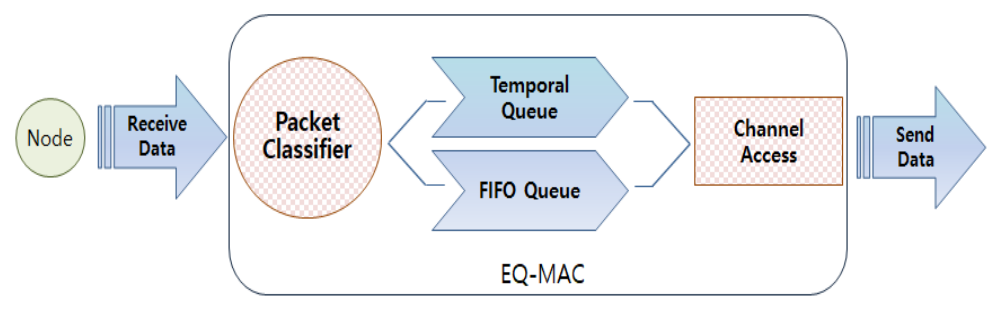

Fig. 1: EQ-MAC operation

\subsection{Q-MAC}

In the Q-MAC structure, QoS is supported by differentiating services based on priority (Zheng et al., 2017). Priority is determined based on the content of the application and the number of traversed hops provided at the MAC layer. It is an advantage to judge data transmission emergency from two points of view, but it does not take into account the surrounding environment of the packet, and thus, does not reflect various network changes. Fig. 2 shows the operation of Q-MAC protocol.

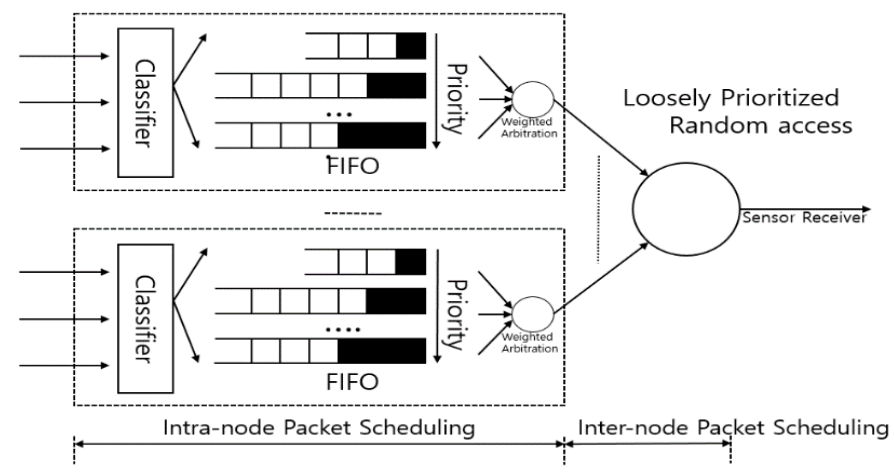

Fig. 2: Q-MAC operation 


\subsection{QAML-MAC}

QAML-MAC is similar to Q-MAC in that the transmission priority varies according to the urgency of the packet, but energy efficiency and delay are improved in the former by changing the transmission order and different schedules of transmitted packets according to their destination. However, the main disadvantage of this algorithm is not highlighting the characteristics of the data generated in the actual network. This is because the algorithm does not reflect the characteristics of the neighboring network or does not have flexibility in transmission when storing the queue.

\subsection{Reinforce learning}

Reinforcement learning is an area of machine learning, where an agent defined in an environment selects an action or sequence of actions that maximizes reward through trial and error in a particular state. In reinforcement learning, we use the carrot and stick method to seek the optimal value. For example, when searching for a maze, if you enter the right direction, +3 points are given, and if you enter a blocked road, -5 points are given. The environment is often built using the Markov decision process. Reinforcement learning is an effective learning method for solving problems with rewards as shown in Fig. 3. It is mainly applied to communication networks, robot control, elevator control, and games such as chess and go.

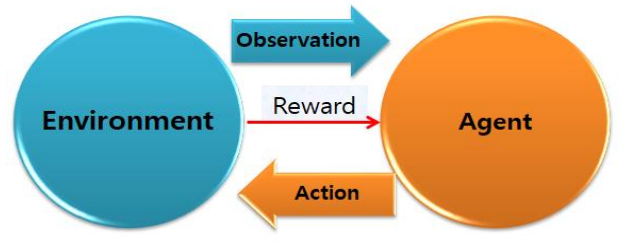

Fig. 3. Reinforcement learning

\section{Protocol Operation Method}

\subsection{Priority Application}

Since the header of the network data packet stores generation information, data length, and destination information, one can know the nature of the data by understanding the contents of the header. To divide input data according to transmission emergency, we proposed a method for classifying the input data into RL Classifier and storing it in a queue. The RL Classifier uses the transmission success rate and transmission failure rate as arguments of the reward function for effective data classification through reinforcement learning. Priority is given differently according to the urgency of the node and the nature of the traffic.

\subsubsection{Agent Reward Value Setting for Priority Classification}

After the data have been collected from the node, they are classified using the information in the header. The information included in the header is the location of 
the data generation and the destination address. The notation for the state probability is as follows. Since the agent operates differently according to the probability value, the input value in the following equation affects the result value of the state probability (Liu and Elhanany, 2006).

$$
V^{\pi}(S)=E_{\pi}\left\{\sum_{k=0}^{\infty} \gamma^{k} r^{k}\left(s_{k}, \pi\left(s_{k}\right)\right) \mid s_{k 0}\right\}=s
$$

In the policy $\pi$, the current state $\mathrm{Sk}$ selects action $\alpha$, the next state is $\mathrm{Sk}+1$, and the expected reward value is E $\pi$. Equation (1) is a function that determines the reward value, and it can be seen that the rewards both in the current state and the policy $\pi$ are affected. Fig. 4 shows the node's reward mark by destination.

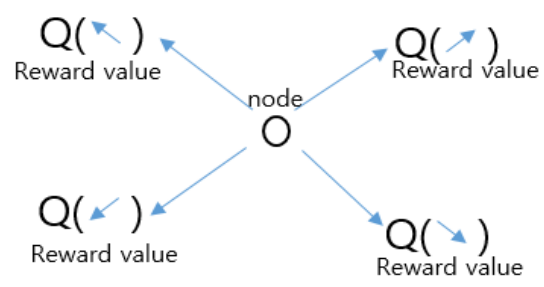

Fig. 4: Node's Reward mark according to destination

\subsubsection{Set Reward Value for Transmission According to Priority}

For normal data with low transmission urgency, Q has a low reward value. The reward value operates as a function that uses an input value as an argument, and reduce the value involved in determining the duty cycle. To determine the transmission probability, the state-action pair is used via Q-learning. Equation (2) shows the Q-value to determine the transmission probability P. R(s, a) is the rewardvalue when action a is taken in state $\mathrm{S}$ (Liu and Elhanany, 2006).

$$
Q^{\pi}(s, a)=R(s, a)+\gamma_{\pi} \sum_{s^{\prime}=S}^{\infty} P_{s s^{\prime}}(a)^{V^{\pi}}\left(s^{\prime}\right)
$$

It is necessary to reduce the duty cycle to reduce energy consumption. DIFS and CW have different lengths according to their priority, thus affecting energy efficient transmission. The higher the priority, the shorter the waiting time, and the lower the priority, the longer the waiting time. Fig. 5 shows the size of DIFS and CW according to priority. 


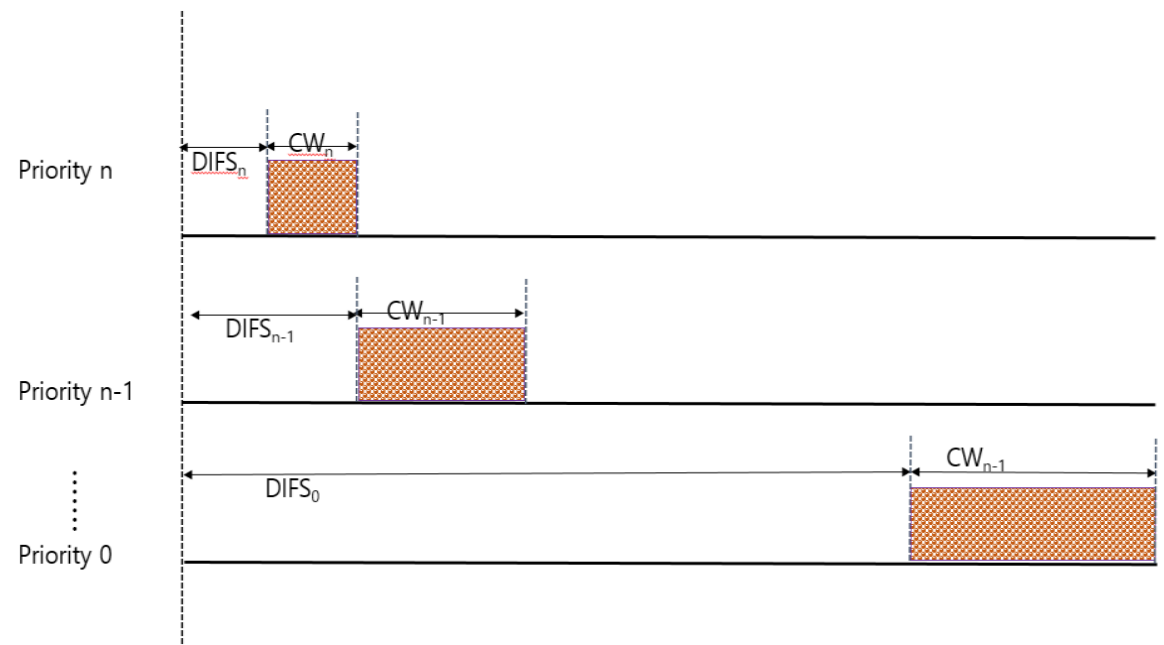

Fig. 5: Example of CW control for transmission according to priority in the existing MAC

In the proposed MAC protocol, the Q-value was adjusted and applied to the duty cycle according to priority. To reduce reward value of the duty cycle transmission probability, the normal data or low priority data was replaced with a value obtained by adjusting the input value variable for Q-value adjustment to a small value. Since event driven data have high priority and must be transmitted first, the Q-value was needed to be increased. Furthermore, the priority application was ensured by adjusting the storage probability of the Q-value. The Q-value with high priority was executed with 1-e probability, and by randomly arranging the probability of e. This operation enhanced the transmission efficiency and guaranteed priority transmission.

\subsubsection{Packet Event Detection and Transmission Policy for Priority Classification}

Normal data are transmitted according to reward values considering energy saving and transmission efficiency.

\section{A. Sudden change in queue size}

If the rate of change in the queue size increases rapidly, it is recognized as an event. When an event occurs in this way, the measured data needs urgent transmission. First, the queue in which transmission is recognized is given a large Q-value of the agent's reward value so that the event data is saved.

\section{B. Sudden changes in measured data}

If the value of measured data changes rapidly, it is considered that an event has occurred. The measured data are then given a high priority.

\subsubsection{Data Classification}

For efficient data classification through reinforcement learning, we experimented as follows.

In this paper, deep learning was employed by dividing it into two parts for data 
classification. The first part was utilized to learn and determine whether the data were normal or event data, whereas the second part was implemented with an algorithm which, when any new data come from learning, can identify whether they are normal or event data. To note, 20,000 input data were tested, and out of that 10,000 were normal data and 10,000 were event data for reinforcement learning.

Deep learning algorithm using Keras was used as a tool, and before and after learning execution was compared. The training data were divided into normal data and event data and entered. Normal data referred to values in which there was a constant interval of time and the range of measurement values was normal. If the value is out of range or the number of measured data suddenly increased and the state changed, it was classified as the event data.

Prior to learning, the data were classified according to the operation of the general QAML MAC protocol, and stored in a queue to check the data transmission rate.

For the pre-training data, the hit rate was less than $30 \%$ for both the event and normal data. As a result of learning 20,000 data classifications, the hit rate increased to $85 \%$ and more. The remaining $15 \%$ were cases where classification was not done properly only for some unusual cases.

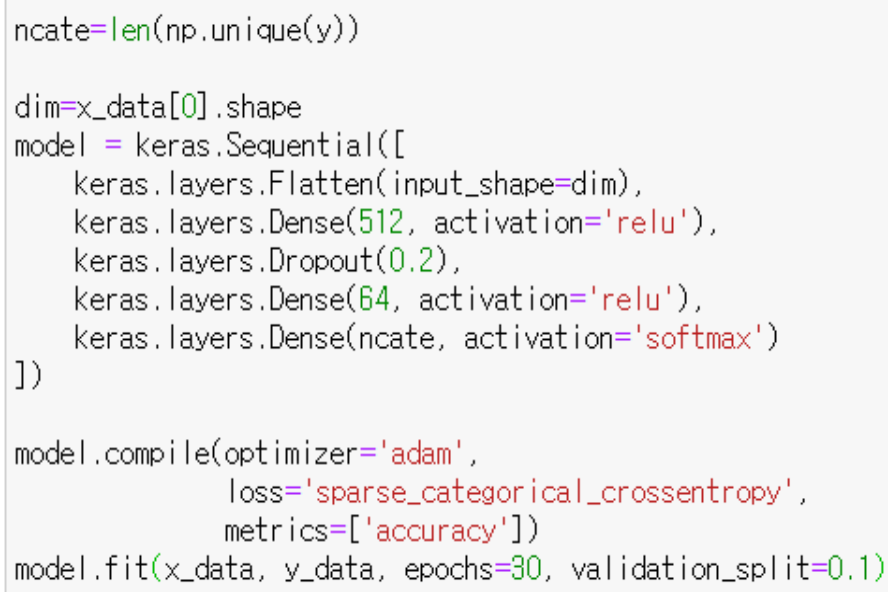

Fig. 6: Deep learning code using Keras

Fig. 6 shows a deep learning code using Keras, an artificial intelligence learning algorithm that determines whether the data are normal or event data. The above code defines the training data consisting of the input tensor and the target tensor, and defines a network consisting of layers that map the input and target. The learning process is set by selecting a loss function, an optimizer, and a measurement index for monitoring. We used fit() which is a method to learn training data. Training data was learned by repeatedly calling fit() method. 
Since transmission data can be predicted through learning, it is possible to quickly classify whether transmission data is normal data or event data. If data classification in the priority queue is performed quickly through prediction, energy waste due to data classification can be reduced and the overall network speed can be improved.

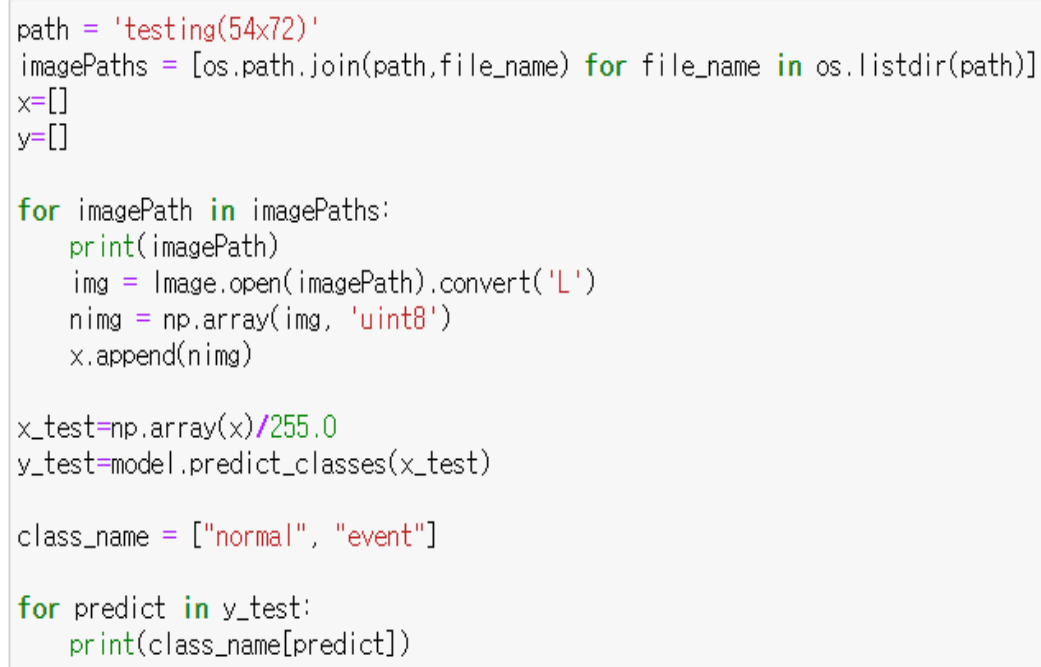

Fig. 7: Data Predict Code

Fig. 7 show a data predict code which can classify as normal or event after learning the two types of data. Use of such predict code improved the efficiency of classification and storage of data.

\subsection{Protocol operation method}

The operation of our proposed protocol is as follows. The collected data are first classified using the reinforcement learning algorithm. Next, the classified packets are stored in queue based on priority. For this, the data input to the node divides the transmission emergency according to the state value of the storage queue, and adjusts the action reward function. The variables that determine the action reward value and state value are decided by the content of the node itself and the traversal value of the MAC layer. Class determination is made by a special classification device called RLClassifier. The RL-Classifier uses artificial intelligence's reinforcement learning algorithm to differentiate data and store it in each queue (Liu et al., 2016 and Park and Kim, 2020). Fig. 8 shows the data storage scheme of the proposed protocol. 


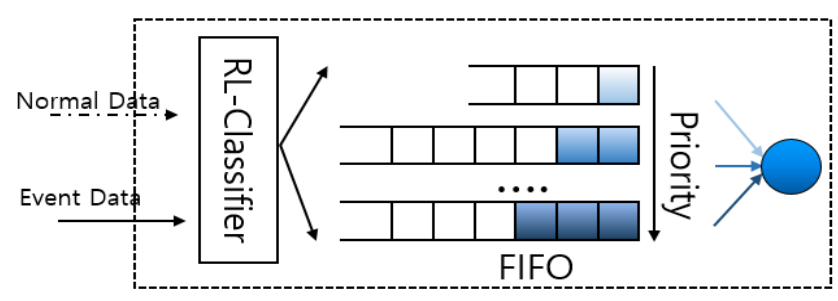

Fig. 8: The proposed MAC operation

Priority is applied to data transfer after the data were stored in the queue. Contention window size is used for priority packet transmission. The contention window size control allows data transmission with different priorities for each class by adjusting the reward value when the window size control function is applied. Fig. 9 shows the diversity of the number of transmission slots based on priority during data transmission. When transmitting the data remaining in $\mathrm{Q}$, the event data get the first priority.
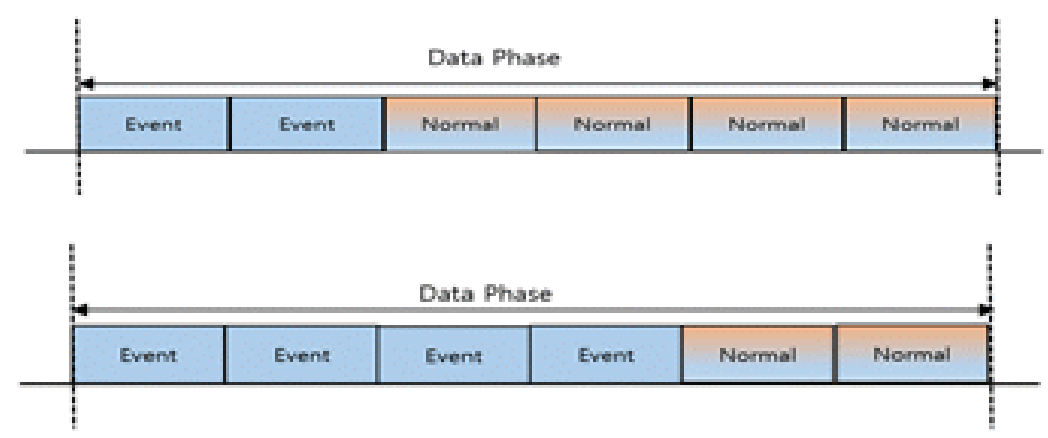

Fig. 9: Various arrangement of slots for priority application

\section{Performance Evaluation}

Simulation was used to demonstrate the superiority of the proposed protocol. Table 1 shows the network and energy parameters of the sensor node used in the simulation. Fig. 10 shows a comparison of delay between the R-QAML MAC protocol and the existing MACs. It can be seen that the average delay is low for data with priority. Since the SMAC protocol does not support priority, the average delay of all the transmitted data is displayed.

Table 1: Simulation environment

\begin{tabular}{|c|c|c|}
\hline Parameter & Description & Value \\
\hline Duty Cycle & & 10 \\
\hline Data_CW & Maximum Window Size & 63 \\
\hline Short_CW & Minimum Window Size & 31 \\
\hline
\end{tabular}




\begin{tabular}{|c|c|c|}
\hline $\mathrm{Pt}$ & Transmit Power Consumption & 0.2818 \\
\hline $\mathrm{Pr}$ & Receive Power Consumption & 0.0 .3682 \\
\hline Pidle & Idle Power Consumption & 0.3442 \\
\hline Psleep & Sleep Power Consumption & 0.00005 \\
\hline Pinit & Initial Energy & 1000 \\
\hline
\end{tabular}

Average Delay with Priority Data

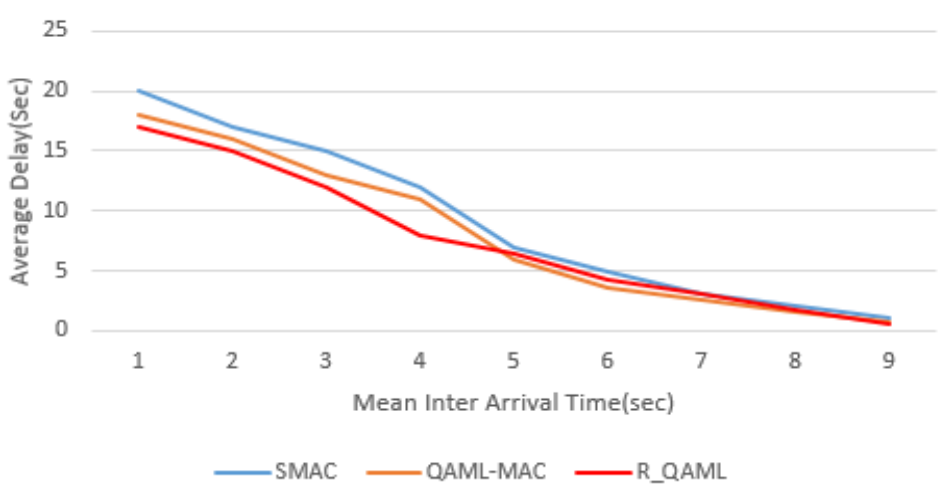

Fig. 10: Comparison of average delay with priority data

\section{Conclusions}

In this paper, to increase efficiency of wireless sensor network, we proposed a new data storage and transmission algorithm using Reinforce learning to store and transmit data according to priorities. It was found that the average packet transmission was smaller than that reported in the past researches. The newly proposed algorithm increased the storage and transmission efficiency by applying the most basic method of artificial intelligence. A study will be conducted in near future on the effects of parameters used on the performance of transmission.

\section{Acknowledgments}

This research was supported by a 2020 Research Grant from SangMyung University.

\section{References}

Alfouzan F., Shahrabi A., Ghoreyshi S. M., and Boutaleb T. (2018). An Efficient Scalable Scheduling MAC Protocol for Underwater Sensor Networks. Sensors (Basel, Switzerland), 18(9), 2806.

Bertsekas D., Gallager R. (1987) Data Networks, Prentice Hall. 
Daehyon K. (2018) Deep Learning Neural Networks for Automatic Vehicle Incident Detection. Asia-pacific Journal of Convergent Research Interchange, SoCoRI, 4(3), 107-117.

Kim H. Y., Kim S. C., and Park H. J.(2018) Priority Aware MAC Protocol for DelayBounded Applications in Wireless Sensor Networks. International Journal of Grid and Distributed Computing, 11(10), 41-50.

Kim S. C., Park H. J., Kim H. Y.(2019) Improving The Network Lifetime of WSN Using An Intelligent Data Collection MAC Protocol. JP Journal of Heat and Mass Transfer Special Issue, 1, 99-106.

Liu Y. et al. (2018). QTSAC: An Energy-Efficient MAC Protocol for Delay Minimization in Wireless Sensor Networks. IEEE Access, 6, 8273-8291.

Liu Z., and Elhanany I. (2006) RL-MAC: A QoS-aware reinforcement learning based MAC protocol for wireless sensor networks. Proceedings of the 2006 IEEE International Conference on Networking, Sensing and control, 06, 768-773.

Mononteliza J.(2020) Research on EIoT Reservation Algorithm Based on Deep Learning. Asia-pacific Journal of Convergent Research Interchange, 6(9), 191-205.

Park H. J.(2011) Energy Efficient MAC Protocol Supporting QoS for Wireless Sensor Networks. Sangmyung Univ. Ph.D.

Park H. J., Kim S. C., and Kim H. Y. (2019) Priority Aware Data Collection MAC Protocol In Wireless Sensor Networks. International Journal of Advanced Science and Technology. 124, 69-78.

Park, H. J. and Kim, S. C. (2020). Design of MAC Protocol for Efficient Data Storage and Transmission Using Reinforce Learning. Journal of Convergence Technologies and Smart Application (JCTSA). HolyKnight, 1, 25-32.

Sri Varsha K., Naga M.R. S. (2018) Applications, Challenges and Protocols of MANETs: A Review. Asia-pacific Journal of Convergent Research Interchange, 4(1), 21-29.

Yahya B., Ben-Othman J. (2010) Energy Efficient and QoS aware medium access control for wireless sensor networks. Concurrency and Computation: Practice and Experience, 22 (10), 1252-1266. 
Ye W., Heidemann J., and Estrin D.(2004) Medium access control with coordinated adaptive sleeping for wireless sensor networks. IEEE/ACM Transaction on Networking, 12(3), 493-506

Zheng G., Sun Y. , Kang B. , Ma H. , Li J. , Wang Y. (2017). A QoS-aware MAC protocol for Wireless Sensor Networks. IJCSI International Journal of Computer Science Issues, 14(1). 